\title{
Soledad en el curso de vida de las mujeres mayores en México y España
}

\author{
Loneliness during life of older woman in Mexico and Spain
}

Fecha de recepción: 12/11/2020

Fecha de aceptación: 07/04/2021

Fecha de publicación: 25/08/2021

https://doi.org/10.48102/if.2021.v1.n2.160

\author{
Rosa María Flores Martínez* \\ rosamariaflores1@gmail.com \\ ORCID: https://orcid.org/0000-0003-2432-124X \\ Doctora en Filosofía \\ Universidad Autónoma de Nuevo León \\ México
}

\author{
Sagrario Garay Villegas** \\ sgarayv@gmail.com \\ ORCID: https://orcid.org/0000-0002-9087-5526 \\ Doctora en Estudios de Población \\ Universidad Autónoma de Nuevo León \\ México
}

\begin{abstract}
* Doctora en Filosofía con orientación en Trabajo Social y Políticas Comparadas de Bienestar Social por la Universidad Autónoma de Nuevo León (UANL). Exbecaria de la Secretaría General Iberoamericana (Segib)-Fundación Carolina. Es profesora e investigadora en la Facultad de Trabajo Social y Desarrollo Humano de la UANL. Sus líneas de investigación son política social, calidad de vida, envejecimiento y vejez, redes de apoyo social, género y familia. ** Licenciada y maestra en Economía por la Universidad Autónoma Metropolitana. Doctora en Estudios de Población por El Colegio de México. Fue coordinadora de la Red de Envejecimiento de la Asociación Latinoamericana de Población del 2010 al 2011 y Secretaria General de esa misma asociación durante el periodo 2017-2018. Ha coordinado diversos proyectos con financiamiento nacional e internacional. Sus líneas de investigación son familia, género, envejecimiento y redes de apoyo sociales. Es miembro del Sistema Nacional de Investigadores, Nivel II.
\end{abstract}

Flores, R. M. y Garay, S. (2021). Soledad en el curso de vida de las mujeres mayores en México y España. Iberoforum. Revista de Ciencias Sociales, Nueva Época, 1(2), 1-30, Artículos, e000160. https://doi.org/10.48102/if.2021.v1.n2.160 Licencia Pública Internacional - CC BY-NC-ND 4.0 


\section{Resumen}

La vejez es considerada como la etapa de la vida en la que puede existir un mayor riesgo de estar solo o sentir la soledad, aunque esta emoción se puede experimentar a lo largo del curso de vida, bordeada por distintas condiciones, matices y significados; asimismo, en la literatura sobre el tema, se ha reportado que la soledad afecta en mayor medida a las mujeres. Por lo anterior, en este artículo, se plantea como objetivo analizar las experiencias de un grupo de mujeres mayores que las han llevado a vivir solas en algunos casos y, en otros, a sentirse solas en distintas etapas de su vida. Con un diseño metodológico cualitativo, se analizan siete entrevistas en profundidad realizadas en México y España. Los resultados revelan que la percepción de soledad se ha presentado en distintos momentos de la vida: en la niñez, frente a la ausencia de hijos o hijas, por la soltería; en cambio, la transición a vivir solas no siempre va acompañada de sentimientos de soledad, incluso se muestra como una soledad deseada, en la que se desarrollan capacidades de afrontamiento, libertad y autonomía.

\section{Palabras clave}

Soledad, vejez, transiciones, curso de vida, género.

\section{Abstract}

Old age is considered the stage of life in which there may be a greater risk of being alone or feeling loneliness, although this emotion can be experienced throughout the life course, defined by different conditions, nuances and meanings; likewise, in the bibliography on the subject, it has been reported that loneliness affects women to a greater extent. Therefore, the purpose of this article is to analyze the experiences of a group of older women that have led them to live alone in some cases and, in others, to feel lonely at different stages of their lives. Using a qualitative methodological design, seven in-depth interviews conducted in Mexico and Spain are examined. The results reveal that the perception of loneliness has occurred at different times of life: in childhood, in the absence of sons or daughters, due to singlehood; on the other hand, the transition to living alone is not always accompanied by feelings of loneliness, and is even shown to be a desired loneliness, in which coping skills, freedom and self-determination are developed. 
Keywords

Loneliness, old age, transitions, life course, gender.

\section{Introducción}

Ante los cambios demográficos y familiares que se han experimentado en las últimas décadas, el fenómeno de la soledad en la vejez cobra relevancia debido, entre otras cuestiones, a las implicaciones en la salud, la calidad de vida y el bienestar de las personas. Si bien es cierto que la soledad no siempre se presenta en las personas de edades avanzadas, es posible que las transiciones experimentadas por las personas mayores (jubilación, viudez, nido vacío) modifiquen sustancialmente sus circunstancias y ello incida en la generación de sentimientos de soledad.

Esta emoción puede experimentarse en diferentes momentos de la vida; no obstante, hay quienes señalan que suele agudizarse en la vejez, pues transiciones vitales como la viudez, la muerte de personas cercanas o familiares, la reducción de la red de apoyo, la jubilación y los cambios en la salud, entre otros eventos, contribuyen a ello (Pinazo y Bellegarde, 2018). Otros estudios han indicado que factores como el estado conyugal, la edad y la salud inciden en la presencia de soledad (Sequeira, 2011; Hacihasanoğlu et al., 2012). Asimismo, se ha mostrado que las condiciones socioeconómicas, el nivel de escolaridad, el rol social, el apoyo familiar, la pertenencia a una red social, las actividades recreativas y el uso del tiempo libre tienen asociación con la presencia de soledad en las personas mayores (Sequeira, 2011).

El sentimiento de soledad puede experimentarse como una emoción o de forma social: la primera suele asociarse con la ausencia de figuras de apego; la segunda se vincula con cambios en el ciclo vital de las personas - por ejemplo, el retiro laboral, la muerte de la pareja, la salida de los hijos e hijas del hogar-, es decir, todo aquello que cambie sus relaciones de convivencia y su rol dentro de la familia o sociedad (Weiss, 1973). Esta segunda forma se asocia, sobre todo, con la soledad objetiva, la cual se refiere a vivir solo (Rubio et al., 2011). Sin embargo, la soledad no es sinónimo de vivir solo o sola: algunos autores señalan que las personas mayores que viven solas suelen mostrar mayores grados de autonomía (Ramos, 2017); en otros casos, representa aislamiento (Martínez y Celdrán, 2019).

También existe la soledad asociada con sentirse solo o sola, la cual se considera una soledad subjetiva - diferente al aislamiento (soledad objetiva)- (Rubio et al., 2011). De acuerdo con Castro Blanco (2010), cuando una 
persona desea estar sola y lo consigue puede ser una experiencia agradable; no obstante, el sentimiento de soledad aparece cuando se quiere estar con otras personas y no se logra o no se tiene con quién estar, es decir, cuando se vive una soledad impuesta (no deseada).

En este artículo, nos centraremos en las mujeres adultas mayores y sus testimonios sobre cómo se sintieron solas en distintos momentos de su vida. Cabe decir que un alto porcentaje de mujeres adultas mayores se encuentran viudas debido a su mayor esperanza de vida, aunque, como se ha mencionado, no necesariamente el vivir solas implica que experimenten soledad.

Este estudio considera dos contextos con panoramas demográficos diferentes que permitirán dar cuenta de cómo se experimenta la soledad en países con un mayor envejecimiento demográfico. Particularmente, se compararán los relatos obtenidos de mujeres mayores provenientes de comunidades rurales de España y México. En el primero de estos países, es más acentuado el envejecimiento de su población: en 2019, el 25.7\% de ésta tenía 60 años o más (Instituto Nacional de Estadística, 2019); mientras que, en México, esta proporción representaba el $12 \%$ en 2020 (Instituto Nacional de Estadística y Geografía, 2020). Adicionalmente, el interés por estudiar los contextos rurales se debe a que estas zonas tienden a un proceso de despoblamiento y envejecimiento que lleva a que las personas mayores se vayan quedando solas (Abellán y Aceituno, 2019).

En México, la región en donde se realizó la investigación fue el estado de Durango. De acuerdo con datos de la Encuesta Nacional de la Dinámica Demográfica (Instituto Nacional de Estadística y Geografía, 2018), esta entidad tiene una población de 1819410 habitantes, de los cuales 51\% son mujeres y $49 \%$ son hombres; con relación al grupo de edad, la mayoría tiene entre 15 y 59 años (61.2\%), le sigue el grupo de 0 a 14 años (26.8\%) y finalmente el grupo que tiene 60 años o más (11.9\%).

Por su parte, en España, el lugar en donde se llevó a cabo el estudio fue la Comunidad de Madrid, que es una de las regiones más desarrolladas del país; cuenta con una alta densidad poblacional, principalmente en las zonas urbanizadas, como consecuencia de la migración de zonas rurales (EpData, 2020). La Comunidad tiene una población de 6663394 habitantes, de los cuales $47.8 \%$ son hombres y $52.2 \%$ son mujeres; al distinguir por grupo de edad, se puede observar que la mayor parte se concentra en el grupo de 15 a 59 años (61.5\%), seguido por el grupo de 60 años y más 
(23.5\%); el grupo de 0 a 14 años es el que tiene la menor proporción (15\%) (Instituto Nacional de Estadística, 2020).

Según el tipo de localidad, en Durango, $68.9 \%$ de la población reside en localidades urbanas, mientras que $31.1 \%$ lo hace en localidades rurales (Instituto Nacional de Estadística y Geografía, 2018). En tanto que, en la Comunidad de Madrid, la mayor parte de la población reside en municipios urbanos (90.9\%); el resto de la población vive en municipios intermedios (8.4\%) y rurales (0.7\%) (Instituto Nacional de Estadística, 2020). Entre ambos contextos existen fuertes contrastes: mientras que, en el caso español (Madrid), hay una proporción más alta de personas mayores (23.5\%), en el contexto mexicano (Durango), el porcentaje es menor (11.9\%); de igual forma, se advierten diferencias según el tipo de localidad: más urbana en Madrid y más rural en Durango.

\section{Algunas miradas sobre la soledad}

Existen diversas formas de aproximarse al concepto "soledad". Por ejemplo, como un estado emocional, ésta se construye dependiendo de las vivencias que cada sujeto le otorgue y de su contexto socio-histórico (Garay et al., 2021). Este tipo de soledad tiene un carácter emocional y suele asociarse con la falta de una figura de apego, el tipo de convivencia cotidiana con otras personas y la participación en redes de apoyo social. Sin embargo, la existencia de relaciones no asegura que la soledad no aparecerá, ya que ello no estriba sólo en el número de relaciones, sino en la calidad de éstas (Castro Blanco, 2010; Cohen-Mansfield et al., 2018; Gajardo Jauregui, 2015; Golden et al., 2009; Heylen, 2010; Pinazo y Bellegarde, 2018; Sequeira, 2011). Esta condición de soledad se vincula, entre otras cosas, a discrepancias entre las relaciones sociales esperadas y las reales, es decir, las expectativas que generan las personas en torno a sus vínculos afectivos y lo que realmente reciben y que consideran insatisfactorio (López y Díaz, 2018).

También existe la soledad social, la cual se refiere principalmente a la pérdida de roles sociales; por ejemplo, la jubilación, la viudez, la salida de los hijos e hijas del hogar, las separaciones o los divorcios. Es decir, cualquier situación que implique una pérdida de contacto con las personas con las que se mantenían relaciones cotidianas y de convivencia (Weiss, 1973). La medición de este tipo de soledad ha tenido varias aproximaciones; una de las más recurrentes es la escala ESTE II, la cual "consta de 15 ítems con tres alternativas de respuesta (siempre, a veces, nunca). Los ítems se agrupan 
en tres factores: percepción de apoyo social, índice de participación social subjetiva y uso de nuevas tecnologías" (Rubio, 2011, p. 528).

En ambos casos, se trata de la percepción que tienen las personas sobre su sensación de relacionarse con otras personas y con su entorno social, la cual puede ser medida a través de técnicas cuantitativas o cualitativas. Además, ambos tipos de soledad están influidos por diversos factores, como la edad, el estado civil, el estado de salud, la condición socioeconómica, la presencia o no de una red social, las actividades de ocio, entre otras (Sequeira, 2011).

Es decir, el sentimiento de soledad (emocional o social) tiene que ver con la soledad subjetiva (loneliness), que se asocia con sentirse solo o sola; ésta es distinta de la soledad objetiva o aislamiento (isolation), es decir, estar solos o solas (Rubio et al., 2011). Esta última se relaciona con la tendencia de una persona a estar sola o aislarse de los demás. Es importante entender la diferencia entre ambos constructos para comprender el fenómeno y las circunstancias que lo rodean, pues que una persona esté o viva sola no necesariamente implica que se sienta sola (Sequeira, 2011; Shiovitz-Ezra y Leitsch, 2010).

Por lo tanto, es posible que personas que viven en el mismo hogar con otros familiares experimenten soledad pues, como se mencionó, depende del tipo de relación que se tenga con las personas con quienes conviven. Inclusive, en algunas investigaciones, se plantea que el uso de nuevas tecnologías - como celulares y computadoras- entre los más jóvenes limita la interacción con sus familiares mayores, provocándoles sensaciones de soledad (Martínez y Celdrán, 2019). De hecho, para Castro Blanco (2010), el sentimiento de soledad más severo es el que se produce estando en compañía.

\section{Soledad y vejez}

Gran parte de las indagaciones en torno a la soledad en la vejez se han centrado en la edad como factor de riesgo; sin embargo, éste no es, necesariamente, un hecho generalizado. Al respecto, Cohen-Mansfield et al. (2018) comprobaron que la soledad no se manifiesta por la edad per se, sino por el aumento de la discapacidad y la disminución de la integración social como factores asociados. Esto quiere decir que la soledad puede presentarse en cualquier momento de la vida y que no está sujeta a una edad particular.

En la literatura sobre el tema, se advierte que la soledad se asocia, por lo regular, con la vejez como si fuese una díada ineludible; sin embargo, 
han surgido otras líneas de investigación que demuestran que esta relación es mucho más compleja y que va más allá de un estado (comúnmente de connotación negativa) que tiene lugar en una etapa de la vida. En estos nuevos estudios, se apunta que no depende únicamente de las circunstancias del contexto en el que se viva, sino también del propio agenciamiento, es decir, "de lo que las personas hacemos y cómo lo afrontamos" (Castro Blanco, 2010).

No obstante, al envejecer, los cambios de roles y la pérdida de familiares y amistades se hacen más frecuentes, lo cual puede provocar que se presenten los sentimientos de soledad en mayor medida durante esta etapa. Como señala Rueda (2018), en relación con las personas mayores y la presencia de soledad, "son personas con limitaciones, suelen tener pocos lazos de relación o lazos débiles, ya sea por perdidas o porque a lo largo de su vida no han fomentado una densa red de relaciones" (p. 3).

En un estudio realizado con personas mayores de España y México, se encontró que las personas españolas presentaban mayores niveles de soledad conyugal; en cambio, la población mexicana mostraba, principalmente, crisis existencial, soledad familiar y social. Estas circunstancias se presentaron sobre todo en las personas mayores sin pareja. Asimismo, las relaciones sociales resultaron más relevantes entre los mexicanos, por lo que la escasa o nula presencia de éstas incrementa sus sentimientos de soledad (Garza-Sánchez et al., 2020).

En otra investigación comparativa entre población de España y Colombia (Rubio et al., 2011), se indica que las personas mayores asocian la soledad con ausencias familiares; por ello, destacan la importancia de la familia como la principal red de apoyo y la relacionan con su calidad de vida. Asimismo, las personas que viven solas muestran mayor soledad en comparación con las que viven acompañadas. Un hallazgo relevante en este estudio es que la percepción negativa de la soledad no se asocia con las condiciones sociales de los países analizados, sino con la vejez.

\section{Soledad, género y vejez}

Los hombres y las mujeres no envejecen igual; las construcciones sociales con relación a los papeles de cada uno determinan la forma en la que lo hacen (Freixas, 1997). Algunos estudios muestran que, para los hombres, las experiencias de vida están estrechamente ligadas con su edad cronológica, pero no ocurre lo mismo con las mujeres: 
En la vida de las mujeres pueden presentarse numerosas combinaciones de profesión-matrimonio-criaturas, con diferentes niveles de temporalización y compromiso que hacen que los roles de esposa, madre y trabajadora puedan adquirir significados diferentes en momentos determinados del ciclo vital, algo que no ocurre en la vida de los hombres, cuya unidireccionalidad de los acontecimientos parece mucho más clara. (Freixas, 1997 p. 33)

Los resultados de algunos estudios evidencian que el proceso de socialización y de asumir roles tradicionales tienen diferencias importantes entre los hombres y mujeres adultos mayores; por ejemplo, se ha visto que las mujeres adultas mayores, al asumir roles tradicionales de cuidado y realización de labores domésticas, suelen ser mejor recibidas en los hogares de sus hijos e hijas para ejercer dichas funciones (Varley y Blasco, 2001; Montes de Oca, 2003). Adicionalmente, se ha observado que, en la vejez, las mujeres adultas mayores reducen sus "obligaciones" familiares (ser esposa, madre y ama de casa), lo cual les genera una sensación de felicidad, mejora su autoestima y su estado psicológico (Freixas, 1997). Al parecer, en la vejez, los hombres se hacen más dependientes y las mujeres más independientes (Freixas, 1997; Yuni y Urbano, 2008). Para las mujeres, los roles de cuidado y afectividad tradicionalmente asignados se mantienen en la vejez y se hacen más importantes para los hombres, lo cual indica, según Freixas (1997), que "los valores de la vejez se hacen, a lo largo de la vida, cada vez más femeninos, mientras se muestran en recesión los más masculinos del trabajo y de la vida pública" (p. 35).

Las diferencias de género mencionadas inciden en cómo las mujeres viven la vejez, así como en su experiencia de soledad. Algunos estudios indican que las mujeres adultas mayores experimentan sentimientos más intensos de soledad emocional, los cuales se agudizan cuando no tienen pareja, tienen problemas económicos o mala salud; mientras que los hombres suelen presentar mayor soledad social (Pinazo y Bellegarde, 2018). Otros estudios muestran que las condiciones socioeconómicas de las mujeres mayores - sobre todo de estratos bajos-, la falta de protección social y la dedicación exclusiva a las labores del hogar y al cuidado de sus hijos e hijas aumentan sus sentimientos de soledad (Rubio, 2011).

No obstante, también se menciona que las mujeres afrontan de mejor manera la soledad en comparación con los hombres (Arroyo y Arreola, 
2021); incluso, se ha mostrado que ellas tienen menos probabilidad de experimentar soledad, particularmente la social, dado que mantienen relaciones afectivas más cercanas con familiares y no familiares (Garay et al., 2021).

\section{Soledad en el curso de vida}

La soledad es una experiencia subjetiva que puede ser percibida en cualquier etapa de la vida; sin embargo, diversas circunstancias asociadas al envejecimiento originan que el vínculo entre la soledad y las personas mayores sea construido socialmente como un binomio inevitable (Castro Blanco, 2010). El fenómeno de la soledad no se vive exclusivamente en la vejez; incluso, es posible que se presente desde la infancia.

Algunos estudios muestran que la soledad se presenta en forma de "U", es decir, que en edades tempranas (adolescentes y jóvenes) es alta, en la adultez disminuye y en la vejez se incrementa; además, los factores que inciden en dicha percepción pueden variar según el grupo de edad que se analice (López y Díaz, 2018; Pinazo y Bellegarde, 2018).

La infancia es la etapa de vida en la cual la identificación de la soledad es más complicada. Durante la niñez, tenemos un vínculo de apego con las personas que nos cuidan; sin embargo, en el caso de que se presente un apego inseguro, éste puede provocar sentimientos de soledad, asociados con la insatisfacción de los vínculos establecidos con los demás, lo que repercutirá en la forma de relacionarnos con otras personas en etapas posteriores (Pinazo y Bellegarde, 2018).

Durante los primeros años de vida, los infantes son partícipes de relaciones afectivas, especialmente con su familia cercana; por lo tanto, la presencia y la calidad del nexo que se genere a través del vínculo parental es esencial para su desarrollo; empero, la ausencia o la falta de calidad de la relación con los padres tienen consecuencias importantes en el desarrollo de los procesos y transiciones posteriores, aunque cabe aclarar que la ausencia parental no siempre se deriva de una omisión familiar: en ocasiones, las carencias económicas o la falta de apoyo social llegan a limitar el establecimiento de vínculos familiares, así como la vida social y personal de los infantes (Martínez y Celdrán, 2019).

La adolescencia se presenta como una etapa llena de contradicciones: por un lado, se busca tener un espacio individual, en el cual se pueda explorar y experimentar; por otro, se demanda la atención de los demás. Es común que, en la adolescencia, se viva soledad; la duración e intensidad 
de ésta dependerá de la calidad y cantidad de las relaciones con sus pares, pues es una etapa en donde al adolescente le interesa ser aceptado y validar sus opiniones con personas de su edad (Qualter et al., 2013).

Las trasformaciones que ocurren en la adolescencia implican la manifestación de cambios biológicos, psicológicos y sociales, muchas veces aparejados con situaciones de crisis, conflictos y contradicciones; la necesidad de adaptación a los cambios que ocurren en esta etapa convierte a la adolescencia en un periodo en donde se crea una nueva identidad, con el riesgo de experimentar sentimientos de separación y responsabilidad, lo que a su vez ocasiona una necesidad más fuerte de la afiliación; de no lograrse, puede repercutir en forma de soledad emocional y social (Carvajal-Carrascal y Caro-Castillo, 2009).

La transición hacia la adultez plantea la construcción de una trayectoria vital que, cimentada en torno a los roles sociales establecidos, marca el curso de vida normativo de una persona adulta (Martínez y Celdrán, 2019). En la adultez, los sentimientos de soledad se asocian con las distintas transiciones que forman parte del ciclo vital de las personas: enfermar, quedarse sin empleo, no tener pareja, no tener hijos e hijas, tenerlos y dedicarse a su cuidado, cambiar de residencia, etcétera. Es decir, se presentan múltiples factores que cada persona asimilará de manera distinta y que, bajo ciertas circunstancias, podría causar soledad, ya sea por un periodo corto o largo de tiempo. Así, durante la adultez, es probable que las personas enfrenten demandas derivadas de los distintos roles (escogidos o impuestos) que se tienen durante esta etapa.

Algunas de estas transiciones se agudizan entre la población adulta mayor, sobre todo las que se refieren a la pérdida de personas cercanas (cónyuge, madre, padre, hijos, hijas) y a los cambios que modifican sus roles socialmente establecidos (la jubilación, el nido vacío). Todos estos eventos transforman la manera en la que se relacionan con su entorno físico y social; por ejemplo, sus redes sociales pueden verse reducidas cuando fallece la pareja o una persona cercana; también, los problemas de salud y la presencia de limitaciones pueden disminuir su satisfacción con la vivienda o con las relaciones afectivas, y podrían acarrearles dificultades familiares (Iglesias de Ussel et al., 2001; Hacihasanoğlu et al., 2012; Pinazo y Bellegarde, 2018).

En términos concretos, de acuerdo con Castro Blanco (2010), la soledad que se vive en la vejez difiere de otras etapas de vida por lo siguiente: 
a) es más una soledad impuesta por las circunstancias que una soledad elegida; b) es una soledad originada por causas duraderas, las cuales son difíciles de modificar, y c) la reducción de las relaciones sociales, con implicaciones cuantitativas y cualitativas. Es importante dejar en claro que estas cuestiones no son las que producen por sí mismas la soledad, sino que representan factores que posicionan a las personas en una situación de mayor vulnerabilidad.

Así, el sentimiento de soledad y la transición hacia vivir solo o sola pueden manifestarse a cualquier edad; no son experiencias que le suceden únicamente a las personas mayores. No obstante, la vivencia de la soledad es distinta en cada historia biográfica, sobre todo por la etapa de la vida en la que se presenta y las circunstancias que rodean a la persona en ese momento, además del propio agenciamiento.

\section{Diseño metodológico y población de estudio}

El objetivo de este artículo es analizar las experiencias que han llevado a un grupo de personas mayores a vivir solas, en algunos casos, y, en otros, a sentirse solas en distintas etapas de su vida. Dado el interés por analizar la soledad subjetiva que experimentan las mujeres en su curso de vida, el diseño metodológico de este estudio es de carácter cualitativo exploratorio. Se realizaron entrevistas a personas mayores residentes de zonas rurales del estado de Durango (México) y de la Comunidad de Madrid (España). El diseño muestral fue por conveniencia; se obtuvo un total de dieciséis entrevistas, de las cuales se utilizaron siete para este artículo debido a que fue ése el número de mujeres que hicieron referencia a experiencias de soledad en su curso de vida. ${ }^{1}$

El criterio para delinear los territorios rurales fue, en un inicio, el tamaño poblacional; específicamente las localidades menores a 2500 habitantes. Sin embargo, dadas las diferencias de los contextos que se están analizando, se recurrió a la conceptualización que plantean Matijasevic y Ruiz (2013), quienes mencionan que la ruralidad no debe reducirse a una cuestión de tamaño poblacional. Por lo tanto, en esta investigación, se con-

1 Las entrevistas se derivan de un proyecto más amplio sobre redes de apoyo en el curso de vida de las personas mayores (Flores, 2020), por lo que los contenidos de las dieciséis entrevistas estuvieron centrados en el análisis de dichas redes y transiciones; sin embargo, entre las categorías emergidas del estudio, se identificaron siete casos de mujeres en donde el tema de la soledad estuvo presente. 
sideró que un territorio es rural cuando "su especificidad es su dependencia de los recursos naturales y su base económica se estructura alrededor de la oferta ambiental en que se sustenta" (Dirven et al., 2011, p. 15). Esta definición considera una nueva forma de relación de densidades poblacionales debido a que incluye concentraciones poblacionales que forman parte de territorios rurales; de igual forma, incluye a todos los sectores económicos que tienen lugar en este tipo de contextos, más allá de las actividades agrícolas (Dirven et al., 2011).

Al inicio del artículo se hizo referencia a los países incluidos en este estudio; ambos comparten idioma y vínculos culturales, políticos, económicos e históricos. A pesar de ello, son lugares muy distintos, no sólo en términos de estructura poblacional, sino en su curso histórico y en las condiciones de vida de las personas mayores (Castillo, 2018). En este artículo, no se pretende comparar los dos países, sino profundizar en las narrativas de las mujeres al considerar su historia local.

En Durango, las entrevistas se realizaron en las localidades de La Ochoa, Veracruz, Narciso Mendoza y Estación Poanas. El acercamiento a la población tuvo el apoyo del Sistema para el Desarrollo Integral de la Familia (DIF Nacional), ubicado en el municipio de Poanas, el cual nos enlazó con las promotoras comunitarias encargadas de atender a las personas mayores de las localidades.

En la Comunidad de Madrid, el acercamiento a las entrevistadas se realizó con el apoyo de la Federación de Asociaciones de Mujeres Rurales (Fademur), a la cual acudían las personas mayores a tomar diversos talleres. Las localidades en donde se realizaron las entrevistas fueron Villarejo de Salvanés, Perales de Tajuña y San Lorenzo del Escorial.

Las mujeres entrevistadas tienen edades en el rango de los 60 a los 84 años. Con relación a su estado civil, la mayoría se encuentran viudas, especialmente las mexicanas; algunas tienen un tiempo prolongado de viudez (40 años), mientras que para otras éste es menor. Respecto al número de hijos e hijas, en México, las personas viudas -aquéllas que estuvieron unidas, generalmente por un periodo extenso- son las que tienen un número elevado de hijos e hijas (entre siete y nueve); en tanto que las solteras y separadas indicaron que no tienen hijos o hijas. Por su parte, en España, independientemente del estado civil de las entrevistadas, el número de hijos e hijas es más reducido (entre uno y dos). 
El arreglo familiar fue diverso; no obstante, en México, el tipo de arreglo monoparental se presentó en mayor medida, derivado del proceso de viudez y de la corresidencia filial de al menos un hijo o una hija; asimismo, dos de las mujeres entrevistadas señalaron que viven en hogares unipersonales. En España, la totalidad de las personas entrevistadas refirieron que sus hijos e hijas no viven con ellas; por lo tanto, ya sea a causa de la viudez o de la emancipación de los hijos e hijas, han conformado hogares unipersonales o, bien, viven con pareja sola o con sus hermanos, hermanas, padres y madres. En este contexto, los hogares son notablemente de menor tamaño, con una tendencia a vivir solas. ${ }^{2}$

Cuadro 1. Características sociodemográficas de las mujeres mayores rurales

\begin{tabular}{|c|l|l|l|l|l|}
\hline País & \multicolumn{1}{|c|}{$\begin{array}{c}\text { Persona } \\
\text { entrevistada }\end{array}$} & Edad & $\begin{array}{c}\text { Estado } \\
\text { civil }\end{array}$ & $\begin{array}{c}\text { Número } \\
\text { de hijos }\end{array}$ & Arreglo familiar \\
\hline \multirow{5}{*}{ México } & Ricarda & 73 años & Separada & 0 & Unipersonal \\
\cline { 2 - 7 } & Martha & 71 años & Viuda & 9 & Unipersonal \\
\cline { 2 - 7 } & Teresa & 69 años & Soltera & 0 & Extensa \\
\cline { 2 - 6 } & María & 84 años & Viuda & 7 & $\begin{array}{l}\text { Monoparental } \\
\text { femenina }\end{array}$ \\
\hline \multirow{2}{*}{ España } & Elena & 60 años & Viuda & 2 & Unipersonal \\
\cline { 2 - 6 } & Lucía & 60 años & Soltera & 1 & $\begin{array}{l}\text { Monoparental } \\
\text { masculina } \\
\text { jefatura paterna }\end{array}$ \\
\cline { 2 - 6 } & Carmen & 63 años & $\begin{array}{l}\text { Unión } \\
\text { libre }\end{array}$ & 2 & Pareja sola \\
\hline
\end{tabular}

Fuente: Elaboración propia con base en las entrevistas realizadas en Durango y Madrid

2 De acuerdo con datos de la Encuesta Continua de los Hogares (ECH) del Instituto Nacional de Estadística (INE), en 2019, del total de hogares unipersonales en España, 41.9\% correspondía a personas de 65 años y más, en su mayoría mujeres (siete de cada diez casos) (Instituto Nacional de Estadística, 2019).

3 Se han cambiado los nombres para mantener el anonimato de las mujeres. 
Precisamente, vivir en hogares unipersonales ha sido uno de los factores de riesgo asociados a la soledad, aunque no necesariamente las personas que lo hacen sienten la soledad, situación que profundizaremos en el análisis. En este sentido, coincidimos con Pinazo y Bellegarde (2018), quienes señalan que la soledad es una experiencia individual y subjetiva que puede manifestarse incluso viviendo acompañado o acompañada.

\section{Análisis de los resultados}

La soledad ha sido un fenómeno generalmente asociado a la vejez; no obstante, puede presentarse a lo largo del curso de vida, en distintos puntos de inflexión y transición dentro de la historia biográfica. Son diversos los factores y circunstancias que pueden llegar a influir en la manifestación de la soledad; en este análisis, nos centraremos particularmente en las experiencias de vida y los significados que se entretejen en torno a ellas. La singularidad de cada caso permite aproximarse a la comprensión de cómo se experimenta la soledad en distintos momentos del curso de vida, desde la subjetividad de las personas.

\section{Las solteras}

Los momentos en los que las mujeres se sienten solas pueden ir entrelazados con la sensación de no estar cumpliendo con las normas socialmente establecidas para ellas. Por ejemplo, la norma social dicta que, en determinado momento de la vida, una persona crea una relación estable con otra persona del sexo opuesto y que los hijos e hijas son el resultado "natural" de esa unión; por lo tanto, el matrimonio y la maternidad son concebidos como "normales" dentro del itinerario de vida (Jelin, 1989). No obstante, no todas las personas cumplen con esta normativa, por lo que, socialmente, su condición es considerada "anómala". Tal es el caso de Teresa: ella no se casó y no tuvo hijos ni hijas; dedicó la mayor parte de su vida al cuidado de su madre en condiciones de dependencia severa: la madre no pudo moverse durante veinte años y, en todo ese tiempo, Teresa fue su cuidadora principal -la apoyaba para realizar actividades instrumentales y básicas de la vida diaria-. Al tener un grado severo de dependencia, su madre requería de apoyo constante; para facilitar el cuidado, dormían en la misma habitación, por lo que Teresa se acostumbró a dormir con ella; cuando su madre falleció, Teresa descubrió que no le gustaba quedarse sola. Ahora enfrenta esta nueva realidad: 
Y le digo a Lupe: "ay, ya no me hallo a quedarme yo sola". Dice: "mira, mira". Dice la Lupe, dice: "mira, mira, pues por eso te hubieras conseguido alguien quien te..." "No", le digo, "estás pendeja". Dice: "pues cómo no te vas a quedar sola, si eres sola". (Teresa, comunicación personal, 14 de julio de 2019)

Según las construcciones sociales en torno al género, Teresa no cumplió con lo que se esperaría del estereotipo femenino (ser madre-ser esposa), aunque, en su vida, el eje rector fue el cuidado de su madre (ser para otros); sin embargo, pese al esfuerzo y el tiempo dedicado a esta actividad, socialmente es una mujer incompleta, puesto que no hay un vínculo que la relacione directamente con un varón; en tal sentido, se le concibe como una mujer carente: "eres sola", le dice una sobrina. Al no tener un marido, un hijo o una hija, su "castigo" es la soledad.

A raíz de un accidente automovilístico, que le ocasionó limitantes físicas, Teresa vive con su hermana, residente del mismo pueblo. Sin embargo, quiere regresar a vivir sola en su casa:

Dice Chela: "no, porque yo me quedo con mucho pendiente" y ya siquiera, dios se lo pague, que, si quiere estar conmigo, a veces le digo: "te serviré de estorbo cuando quieres salir a alguna parte, ya ni vas a las peregrinaciones", pero le digo: "no te preocupes, déjame a mí, qué le hace que esté sola"; dice: "pues sí, pero no". Dice ella: "hasta que yo te vea que tú solita"; le digo: "no esperes que yo solita, voy a batallar, voy a dilatar" digo porque mis pieses yo no los siento bien, los siento como dormidos así es de que mientras el doctor no me dé de alta... y luego dice ella: "pues pa' que veas; mientras no te dé de alta, tú no te vas de aquí", aunque como sea dice: "deja que te dé de alta". (Teresa, comunicación personal, 14 de julio de 2019)

Un caso similar es el de Lucía, quien, aunque cumplió con el rol de la maternidad, no lo hizo en pareja. La maternidad es un proceso valorado por la sociedad; sin embargo, cuando sucede al margen de las normas sociales y preceptos de corte tradicionalista y patriarcal, ratificados en el rito del matrimonio, es objeto de vigilancia y sanción familiar y social. La decisión de Lucía de asumir la maternidad en su calidad de "soltera" generó conflictos al interior de su familia: 
O sea que yo quiero tener mi niño, no funcionó la relación, pero me da igual, pero este niño viene y... yo me pasé el embarazo porque rompí con... con este chico y me volví a España embarazada de cuatro meses, mis padres me encerraron en casa. El resto del embarazo yo no podía salir a la calle, no podía hacer ni llamadas telefónicas, no... podía... enterarse nadie, ni los familiares ni nadie, no. (Lucía, comunicación personal, 29 de noviembre de 2019)

En estas condiciones -y dadas las asimetrías de poder al interior de la familia-, los padres fueron quienes tomaron decisiones respecto a la forma en la que Lucía vivió su embarazo: aislada, oculta y en soledad. Si bien Lucía encontró protección en su red familiar para continuar su embarazo, la ayuda estuvo condicionada por el castigo y la sanción moral, cristalizándose en actos de violencia y aislamiento.

El embarazo como punto de inflexión en su vida, así como las circunstancias adversas que rodearon el proceso al interior de la dinámica familiar, han tenido repercusiones a lo largo de su vida, pues propiciaron la ruptura de lazos familiares y relacionales. Lucía fungió como cuidadora principal de su madre, quien padecía Alzhéimer en etapa avanzada y posteriormente falleció; ahora, tiene la expectativa de otorgar cuidados a su padre, en caso de ser necesario, como parte de un deber filial, pese a la inexistencia de vínculos afectivos:

Digamos que el lazo afectivo como que se rompió, yo no les quiero, a mis padres, o sea, a lo mejor soy un monstruo, no. Ese lazo afectivo se rompió, no se ha construido una relación sana a lo largo de los años, pero yo les voy a cuidar hasta el final. (Lucía, comunicación personal, 29 de noviembre de 2019)

Desde su embarazo, el aislamiento y la escasez de relaciones más allá del grupo familiar han sido una constante en su vida. Al preguntarle sobre sus amistades, señala: "No, no, no conservo, no conservo a casi nadie". No obstante, ha encontrado actividades que disfruta realizar en soledad, como la lectura y la fotografía: "Yo tengo mi habitación, con mis libros y mis cosas"; los momentos en los que realiza estas actividades los significa como espacios de refugio y tranquilidad. 
En los relatos mostrados, se puede observar que, en el caso de Teresa (México), su condición de soltería y cuidadora de su madre a lo largo de su vida le genera sentimientos de soledad en su vejez, lo que la lleva a decir que no se "halla a quedarse sola"; sin embargo, su relato se muestra un tanto contradictorio porque quiere vivir sola, al parecer para dejar de ser una carga para su hermana. En cambio, en el caso de Lucía (España), ser madre soltera la llevó a enfrentarse a cuestionamientos familiares y sociales y a romper lazos afectivos con sus padres; sin embargo, en su relato, deja entrever, más que sentimientos de soledad, cierto aislamiento. La vulnerabilidad que enfrentan las mujeres que no cumplen con las expectativas sociales en torno al matrimonio puede traducirse en estigma, aislamiento y autoidentificación de los estereotipos derivados de la presión social (Martínez y Celdrán, 2019).

\section{Las viudas y separadas}

La viudez, como punto de inflexión en la vida de las mujeres, puede llegar a ser significado de manera distinta según el momento en el que ocurra durante el curso de vida, la situación familiar, el género y los recursos con los que se cuente, entre otros aspectos. Para Elena, la muerte de su pareja sucedió en una etapa temprana en el ciclo familiar -durante la infancia de sus hijos e hijas-y en medio de conflictos conyugales:

Miguel y yo nos conocimos en el año 88, 89, 87 quizás, 87, sí, nos casamos en el momento y nacieron los niños en el 92, creo que fue así, en el 92 y entonces eso ha sido otra, otra circunstancia para fijar mi residencia aquí. Más tarde, bueno, mis hijos son gemelos, más tarde, mis hijos fueron creciendo. Miguel y yo nos llevábamos bastante mal, los regañábamos mucho, pero bueno ahí seguimos juntos y Miguel falleció en el año 2002. Y bueno, pues afronté la... de llevar las cosas yo, en primer... Mis hijos ya tenían entonces 10 años, no eran grandes pero bueno, todavía, todavía... eran pequeños, sí, pero bueno ya tenían lo más difícil, la infancia ya estaba pasando, a los 6 años es una etapa más difícil, eso ya estaba pasando y desde entonces voy haciendo... voy dirigiendo mi vida en solitario y sin... Eh, bueno, en libertad. (Elena, comunicación personal, 29 de noviembre de 2019) 
La viudez femenina se asocia comúnmente con aspectos de carácter negativo, en gran medida por el orden patriarcal y el posicionamiento social de las mujeres; dado que la identidad femenina suele definirse desde la figura masculina, la pérdida del cónyuge supone una situación de indefinición y desamparo (Galicia et al., 2020). No obstante, Ramos (2017) introduce otra mirada cuando señala que las mujeres, dadas las situaciones que han vivido a lo largo de su vida, desarrollan una capacidad de afrontamiento que les permite asumir y agenciar los cambios de una mejor manera. En el caso señalado, la diada formada por la viudez y la soledad fue afrontada por Elena desde un significado de libertad.

Ahora bien, Martha, quien quedó viuda cuando tenía 64 años, recuerda, desde la distancia temporal, la etapa previa al fallecimiento de su esposo como un proceso complejo: por una parte, la sobrecarga de fungir como su cuidadora principal por un periodo de tres años - él tenía cáncer- afectó su estado físico y psicológico; por otra parte, debido a su temor a quedarse sola, deseaba que su pareja no falleciera y continuar cuidándolo, pese al deteriorado estado de salud de su cónyuge enfermo y de ella como cuidadora. “QQue no se muera, yo lo navego, que no se muera, que no se vaya!", pensaba.

Sobre la etapa previa a la viudez, rememora:

Yo andaba toda mal, muy desvelada, me quedaba dormida, me quedaba dormida, si estaba platicando me quedaba dormida. Yo decía: "ique no se muera, yo lo navego, que no se muera, que no se vaya!". Yo no quería estar sola, yo no quería quedarme sola, yo quería que él viviera. (Martha, comunicación personal, 3 de enero de 2019)

Lagarde (2014) señala que "las mujeres son construidas y viven sus vidas como seres incompletos y cautivos" (p. 789); de ahí que, para existir, deban buscar su continuidad en los otros: los padres, los cónyuges, los hijos e hijas, la casa o la causa; de tal forma que, en este mirarse en los otros, el curso de vida de las mujeres se transita en el acatamiento del ser femenino para-los-otros y como entes cautivos de-los-otros.

Para quienes llegan a edades avanzadas, la probabilidad de experimentar transformaciones en la estructura familiar es alta pues, al igual que el individuo, la familia tiene su propio curso. En ese sentido, el fallecimiento de alguno de los miembros impacta no sólo en los arreglos residenciales, sino también en los vínculos y en los estados subjetivos de las personas. 
En los hogares en donde correside únicamente la pareja, al fallecer uno de los miembros, la experimentación de soledad no deseada es un efecto recurrente. La pareja representa una fuente de apoyo clave al final de la vida, asociada con el bienestar, la compañía y la cercanía física y emocional (Scott y Wenger, 1996). Por lo tanto, el deceso representa una pérdida significativa, que posiciona a las personas en una situación de vulnerabilidad, al mismo tiempo que desequilibra la estructura familiar y genera soledad (Montes de Oca, 2011).

El temor a la soledad debido a la viudez es un estado recurrente entre las personas mayores casadas que han estado fungiendo como cuidadoras principales de sus parejas, especialmente después de transitar por largos procesos de enfermedades crónicas o estados de dependencia. Las necesidades y demandas de apoyo permanente absorben gran parte de su tiempo y energía, de manera que, paulatinamente, centran tanto su vida en las personas que cuidan que se produce un aislamiento de otros vínculos sociales y familiares.

En retrospectiva, después de la viudez, Martha narra:

Yo me sentía triste. Pero ya después como que me fue entrando como la resignación y se me fue quitando el peso, asina, y ya después como así que... que ahora que diga que le echo de menos ya no, ya no, ya me siento como que me libré, así ya, ya no... ya no me siento con... es que fue una carga pesada. (Martha, comunicación personal, 3 de enero de 2019)

Si bien la muerte del cónyuge dependiente se percibe como una pérdida significativa, también representa una liberación: las personas cuidadoras comienzan a reorganizarse y reapropiarse de su vida. Martha fue resignificando el proceso: "me fue entrando como la resignación", "se me fue quitando el peso". Su temor a la soledad ya no está presente; cuando dice "ya no me siento con...", se refiere a que ya no se siente con tristeza ni soledad; los deberes de cuidado fueron una carga pesada de la cual quedó liberada.

Cuando ocurren eventos como la viudez, las familias suelen acoger a la persona que sufrió la pérdida conyugal, sobre todo en el caso de las mujeres mayores; éstas son bien recibidas en el hogar de los hijos o hijas porque, generalmente, contribuyen con las actividades domésticas y el cuidado de los nietos y nietas (Varley y Blasco, 2001). Aunque las personas que 
han enviudado no siempre desean adherirse a otro núcleo familiar, cuando se trata de las mujeres se asume, socialmente, que no pueden vivir sin un hombre. La percepción de los hijos varones adultos sobre sus madres como mujeres frágiles y dependientes es un reflejo de los estereotipos de género que limitan las decisiones de las mujeres, aun cuando éstas sean mayores que ellos y cuenten con sus propias percepciones de su individualidad:

No, no, yo le decía que se fuera, yo veía a la mujer que no estaba a gusto. Él decía: "yo no me voy, ¿cómo la voy a dejar sola?". "No, mijo, a la hora que se queran [quieran] ir, yo no me hago nada en mi casa, yo estoy bien". Duermo bien, como bien, bendito sea dios que tengo que comer, no batallo por comida, ya uno u otro me manda y pos dios me socorre. "No tenga pendiente". (Martha, comunicación personal, 3 de enero de 2019)

Tanto para Elena (España) como para Martha (México), la viudez significó enfrentarse a una situación para la cual no estaban preparadas por sus roles sociales. A pesar de los conflictos o desgaste por el cuidado que les implicaba tener una pareja, habían sido socializadas para estar en matrimonio y es probable que, por ello, la transición les resultara difícil de asimilar. En el primer caso (Elena), por la etapa del ciclo familiar en la que se encontraba; en el otro (Martha), por la sensación de ser-para-los-otros y de repente romper con esa dinámica. Sin embargo, ambas afrontan la situación a través de diversos mecanismos que les permiten eliminar ese sentimiento de soledad o tristeza en esta etapa de su vida.

Las mujeres separadas enfrentan una realidad un tanto diferente a la de las viudas: para las segundas, haber perdido a su pareja no supone una elección; para las primeras, separarse pudo ser su decisión. Lo anterior puede ocasionar cuestionamientos sociales y de la propia familia por "estar solas", los cuales parecen agudizarse en la vejez. Ricarda, por ejemplo, vivía con su hermano en la casa heredada por sus padres; al fallecer él, se quedó sola. En su caso, se han activado otras redes de apoyo familiares: su hermana, por ejemplo, le ofreció cambiarse de residencia; sin embargo, Ricarda expresa su desacuerdo en aceptar este apoyo a través de la metáfora "en casa ajena el muerto y el arrimado... al rato [...] apesta". Ricarda percibe que, al habitar en una vivienda que no es la propia, a corto o largo plazo, su 
presencia generará molestia y sufrimiento, por lo que prefiere evitar este malestar y continuar en su hogar unipersonal.

Nomás que se acaba uno, ya quedé sola. Querían que me vaya. No. En otra casa, arrimada uno no, peor que le hacen mala cara, y otro día no, no es igual. Pero en casa ajena el muerto y arrimado... al rato, cómo, cómo dice... apesta, pos así la palabra... un día ven buena cara y otro día ya no, qué anda haciendo uno, sufriendo. No le digo, esa muchacha que está retratada me dice: "vámonos; qué estás haciendo aquí sola". Noo, luego vienen y me buscan, me buscan. No, si allá duré mucho, allá con Lucina. (Ricarda, comunicación personal, 6 de julio de 2019)

Al final del fragmento, señala que ya experimentó anteriormente vivir por un tiempo prolongado con su hermana; esta vivencia le da elementos para confirmar que su metáfora es cierta. Aunque el caso de Ricarda parece un caso aislado, en realidad es un fenómeno creciente, especialmente en los países desarrollados. ${ }^{4} \mathrm{Al}$ estado conyugal de Ricarda se le suma no haber tenido hijos o hijas durante su unión, motivo que, aparentemente, desencadenó su separación. La infertilidad en una mujer unida se considera sumamente cuestionable, pues va en contra del orden "natural". La incapacidad de engendrar, por ende, se percibe como una grave falta que incide en la construcción de una imagen negativa en torno a las mujeres sin hijos.

Según lo narrado por Ricarda, se unió en matrimonio "ya grande": tenía aproximadamente 35 años cuando se casó; su cónyuge tenía la misma edad. La duración de su matrimonio se limitó a siete años. Antes y después de la unión, su pareja migraba de manera constante y por ciertos periodos a Estados Unidos; sin embargo, en una ocasión, ya no regresó: "se fue y me dejó", dice. De acuerdo con la narrativa, la causa de su separación fue su imposibilidad para engendrar:

4 En México, de acuerdo con la Encuesta Nacional de Hogares 2017, el 88.6\% de la población vive en hogares familiares; no obstante, la proporción de hogares unipersonales ha tenido un ligero aumento, especialmente en las entidades de Quintana Roo (18.4\%) y Ciudad de México (15.3\%) (Instituto Nacional de Estadística y Geografía, 2017). Residir en hogares unipersonales se configura como una tendencia entre los jóvenes, aunque, con el acelerado proceso de envejecimiento poblacional en el país, es posible que, en las próximas décadas, haya un incremento de este tipo de hogar entre la población, como ha sucedido en otros países.

Flores, R. M. y Garay, S. (2021). Soledad en el curso de vida de las mujeres mayores en México y España. Iberoforum. Revista de Ciencias Sociales, Nueva Época, 1(2), 1-30, Artículos, e000160. https://doi.org/10.48102/if.2021.v1.n2.160 Licencia Pública Internacional - CC BY-NC-ND 4.0 
Yo no supe tener y ya... [silencio]. Pos ni modo, a veces digo yo "qué bueno que no tuve, ahí anduviera navegando con la familia, como quiera aquí sola ni quién". Por eso se fue y me dejó. No, pos si nomás por tantearse a la gente, pero pos lo vía [veía] yo bien, nomás duramos siete años. Ay, tan bonitos los niños. Yo que no serví... para... [silencio]. No tuve yo nada, por eso se fue el hombre y me dejó, se fue y ya no volvió. Mario se fue [silencio], ya no volvió. (Ricarda, comunicación personal, 6 de julio de 2019)

Aunque la narrativa gira en torno a la no procreación de hijos, la palabra no es pronunciada; por lo tanto, se advierte que es un tema difícil de expresar. Además, particularmente en este fragmento, hubo varios momentos de silencio que Ricarda utilizó como una estrategia discursiva para enfatizar lo doloroso que le resulta hablar del tema. Concibe el cuerpo como un instrumento de creación; no obstante, ante su incapacidad reproductora, lo significa como un objeto inservible que no fue capaz de concebir. En su discurso, asume completamente esa responsabilidad: fue ella la que no tuvo hijos. La consecuencia de ello fue que el cónyuge, en su posición de varón, tomara la decisión sobre ella: "me dejó"; a partir de este evento, surgen sentimientos de abandono, culpa y soledad.

El núcleo fundante de la identidad femenina se asocia con el ser madre, de manera que quien no lo es sufre cuestionamientos y es objeto de presiones sociales. Dentro de los "saberes" y "deberes" naturalizados como parte del ser mujer, el principal es ser madre. La incapacidad de parir hijos se traduce en la negación de uno de los eventos constitutivos de las mujeres: la procreación; de ahí que la esterilidad en las mujeres esté marcada por la culpa y el castigo (Lagarde, 2014). Para Ricarda, el castigo fue el abandono de su cónyuge.

\section{La enfermedad}

Ante una situación de deterioro en la salud, no todas las mujeres mayores cuentan con apoyo; en las experiencias de Carmen y Lucía, se narra la falta de apoyo filial, lo que evidencia que el hecho de tener hijos no es un determinante que asegure contar con soporte y compañía en casos de enfermedad o necesidad de atención médica.

En la familia de Carmen, no todos los miembros brindan apoyo en la misma medida; asimismo, quien lo recibe tiende a percibirlo de distinta 
forma. En este caso, existen diferencias de género: mientras que con la hija se mantiene una relación cercana, con el hijo varón hay un mayor distanciamiento, mediado por el apoyo y acompañamiento dedicado al padre.

Ellos van con su padre. Mi hija no, pero a Manuel sí le echo de menos, le digo: ijolines!, a su padre sí lo acompaña a todos lados y a mí no ha sido capaz de decirme "te acompaño, mamá", no, no, no. Yo digo bueno, ya bastante tienen con su padre, porque también la está pasando mal, así que... (Carmen, comunicación personal, 14 de noviembre de 2019)

Culturalmente, se da por hecho que, cuando las madres envejecen o enferman, los hijos e hijas les brindarán apoyo como una forma de correspondencia y reciprocidad por los cuidados recibidos; no obstante, no es una situación generalizada, especialmente en contextos en donde hay bajas tasas de natalidad o una alta frecuencia de procesos migratorios de la población joven, como sucede en las zonas rurales de España.

Me ha tocado estar varias horas, no me voy yo sola, llamo una ambulancia, me voy yo sola. Y... este año pasado me acompañó mi hijo porque estaba aquí y se vino conmigo, llamamos a un taxi porque la ambulancia iba a tardar mucho. Llamamos a un taxi y se vino conmigo él, pero si no me voy yo sola, llamo la ambulancia y me voy yo sola. (Lucía, comunicación personal, 29 de noviembre de 2019)

En la Comunidad de Madrid, se cuenta con servicios de traslado médico que, aunque no sustituyen la compañía familiar, posibilitan la atención. En el caso de Lucía, dado su estado de salud deteriorado (presenta varias enfermedades), es habitual que requiera atención sanitaria; al estar sola, enfrenta la necesidad de resolver por sí misma el traslado y la estancia en el hospital.

\section{Conclusiones}

La soledad es un fenómeno complejo, de carácter multidimensional, que se vive de manera diferenciada según el momento de la vida en el que se presente. A lo largo del curso de vida, se puede llegar a experimentar el sentimiento de soledad, el cual puede estar vinculado con aspectos como 
la edad, el género o el hecho de vivir solo o sola; sin embargo, éstas no son condiciones que lo determinen por sí mismas.

Uno de los virajes más significativos en la vida de una persona es la muerte de algún familiar con un vínculo cercano; por ejemplo, cuando muere la pareja, se modifican las relaciones y el desarrollo de otras trayectorias que trascienden a lo largo de la vida. Estos eventos pueden ocurrir en distintas etapas. No obstante, en la vejez, este cambio se puede agudizar porque confluye con eventos que pueden hacer más conflictivo asumir el estado de vivir solo o sola. Es importante mencionar que, para algunas mujeres mayores, tanto la muerte de la pareja como encontrarse solas se resignifica como un proceso liberador, especialmente después del otorgamiento de cuidado de largo plazo.

Las narrativas compartidas en el estudio indican que, durante el desarrollo del curso de vida, gran parte de las experiencias vividas por las mujeres se encuentran ligadas al cuidado de los otros por un tiempo prolongado. Al dedicar un periodo amplio de su vida al bienestar de la persona cuidada, se aíslan de la vida social y comunitaria, disminuyen el contacto con otras personas y, en la mayor parte de los casos, se olvidan del cuidado de sí mismas. La convergencia de estas situaciones repercute en el desarrollo de la soledad femenina en compañía de la persona cuidada, que es, posiblemente, una de las formas más duras de sobrellevar.

Nuestro estudio pone en relieve que la soledad no tiene una edad fija de aparición; por lo tanto, no es exclusiva de la vejez. Aunque es importante matizar que, en dicha etapa, la soledad presenta expresiones singulares, pues se combina con otras situaciones -fallecimiento de familiares, enfermedades crónicas, dependencia, fragilidad, cuidados- que complejizan su afrontamiento. No obstante, esto no significa que no pueda ser superada e inclusive deseada pues, en algunos casos, se ha llegado a concebir como un proceso previo a la obtención de una mayor autonomía y libertad.

Otro de los hallazgos apunta a que el género es un aspecto medular en la comprensión de la soledad, debido a que tiene implicaciones en las distintas y diversas experiencias compartidas por las mujeres mayores en torno a esta cuestión. Consideramos que esto se vincula con la construcción identitaria en función del orden social genérico de edad, pues existen expectativas, necesidades, limitantes y oportunidades marcadas por el género y la edad, con un impacto significativo en las condiciones de vida de las mujeres. 
Como dice Lagarde (2012), a las mujeres "nos han enseñado a tener miedo a la libertad, a tomar decisiones y a la soledad" (p. 198). Lo anterior limita nuestra autonomía y capacidad de decisión a lo largo de nuestra vida pues, incluso, socialmente, se piensa que las mujeres debemos estar "acompañadas", lo cual nos posiciona como seres infantiles, incapaces de asumir nuestra soledad (p. 200). Esta última concepción se refleja en los relatos de varias adultas mayores que desean estar solas pese a que no se les permite hacerlo, en parte como un elemento de protección social, aunque también porque, en el imaginario de las familias, parece que esto acarrearía soledad para las mujeres.

La forma de construirnos a lo largo de la vida es un elemento fundamental que determina cómo experimentaremos nuestra vejez; en el caso de las mujeres mayores, hemos encontrado relatos que denotan independencia y capacidad de resiliencia para enfrentar los cambios que han acaecido durante su curso de vida. El análisis comparativo no muestra diferencias sustanciales en los contextos y las experiencias de soledad; queda claro que las construcciones sociales en torno a lo que significa ser mujer han incidido en el curso de vida de las entrevistadas y en los momentos que han experimentado sentimientos de soledad, al parecer más por una carga socialmente impuesta que no les permite verse como seres individuales y que las lleva a definirse en función del otro; por ejemplo, la mayoría experimentó soledad por no saber qué hacer ante la pérdida del otro: padre, madre, esposo, hijos, hijas. Sin embargo, también mostraron su capacidad para afrontar la soledad y expresar sus deseos de vivir solas sin sentirse solas.

En general, se advierte que son múltiples las emociones derivadas de las experiencias de soledad, lo cual es un reflejo del entrelazamiento de afectos y conflictos, deseos e imposiciones, posibilidades y limitantes, soledad y compañía. En este complejo entramado, surgen sentimientos de tristeza y liberación, de miedo y resignación, abandono y culpa, deberes y disidencias; en algunos casos, de manera opuesta; en otros, convergiendo en un mismo momento.

Por todo lo anterior, se plantea que el fenómeno de la soledad debe analizarse desde distintas aristas; hace falta, principalmente, explorarlo desde las subjetividades, ya que cada persona lo puede experimentar de distinta manera, según el momento en el curso de vida, el evento que lo detone, las circunstancias familiares, el género, la situación emocional y física, entre otras cuestiones. 
Finalmente, planteamos que es necesario fomentar la visibilización política de la soledad no deseada; para ello, es preciso dejar de concebirla como un asunto individual y privado. De no atenderse, las consecuencias pueden llegar a afectar de manera objetiva y subjetiva el bienestar y la calidad de vida de las personas de distintos grupos poblacionales, incluidas las personas mayores. Asimismo, enfatizamos que el establecimiento de políticas sociales respecto a esta cuestión será fundamental para su prevención, pues la soledad no es un estado invariable, sino que puede ser afrontado y transformado.

\section{Referencias bibliográficas}

Abellán, A. y Aceituno, P. (2019). Los escasos, dispersos y envejecidos electores de la España rural. Envejecimiento en red. http://envejecimientoenred.es/los-escasos-dispersos-y-envejecidos-electores-de-la-espana-rural/

Arroyo, C. y Arreola, L. (2021). Viviendo la soledad. Significados y motivaciones de hombres y mujeres mayores. En C. Arroyo (coord.), Las soledades en la vejez. Experiencias, significados y afrontamientos (pp. 31-56). Universidad Juárez del Estado de Durango.

Carvajal-Carrascal, G. y Caro-Castillo, C. V. (2009). Soledad en la adolescencia: Análisis del concepto. Aquichan, 9(9), 281-296.

Castillo, R. (2018). Los modelos de cooperación cultural de México y España a través de sus institutos culturales, desde una perspectiva para el desarrollo [Tesis de maestría]. Instituto de Investigaciones Dr. José María Luis Mora. https://mora.repositorioinstitucional.mx / jspui/bitstream/1018/422/1/Ricardo\%20Castillo_Modelos \%20 M\%C3\%A9xico \%20Espa \%C3\%B1a.pdf

Castro Blanco, M. P. (2010). Soledad y envejecimiento: Cómo evitar que este binomio se cumpla. En Hartu-emanak, Las personas mayores en el umbral del siglo XXI. X Jornadas. La autonomía personal y la dependencia en el proceso del envejecimiento (p. 33-45), Hartu-emanak, Diputación Foral de Bizkaia, BBK. https://www.hartuemanak.org/ wp-content/uploads/2015/11/Publicacion10.pdf

Cohen-Mansfield, J., Hazan, H., Lerman, Y., Shalom, V., Birkenfeld, S. y Cohen, R. (2018). Efficacy of the I-SOCIAL intervention for loneliness in old age: Lessons from a randomized controlled trial. Journal 
of Psychiatric Research, 99, 69-75. https://doi.org/10.1016/j.jpsychires.2018.01.014

Dirven, M., Echeverri Perico, R., Sabalain, C., Rodríguez, A., Candia Baeza, D., Peña, C. y Faiguenbaum, S. (2011). Hacia una nueva definición de "rural" con fines estadísticos en América Latina. Comisión Económica para América Latina y el Caribe. https://www.cepal.org/es/publicaciones/3858-nueva-definicion-rural-fines-estadisticos-america-latina

Doblas, J. y Conde, P. (2018). El sentimiento de soledad en la vejez. Revista Internacional de Sociología, 76(1), e085. https://doi.org/10.3989/ ris.2018.76.1.16.164

Dykstra, P. A. y Jong Gierveld, J. de (2004). Gender and marital-history differences in emotional and social loneliness among Dutch older adults. Canadian Journal on Aging. La Revue canadienne du vieillissement, 23(2), 141-155. https://doi.org/10.1353/cja.2004.0018

EpData (2020). Población en España hoy: Inmigrantes, emigrantes y otros datos sobre los habitantes de España. https://www.epdata.es/datos/ poblacion-espana-hoy-inmigrantes-emigrantes-otros-datos-habitantes-espana/1/espana/106

Flores, R. M. (2020). Vejez rural, trayectorias familiares y redes de apoyo en el curso de vida: Un estudio comparativo entre México y España [Tesis doctoral]. Universidad Autónoma de Nuevo León.

Freixas, A. (1997). Envejecimiento y género: Otras perspectivas necesarias. Anales de Psicología, 73, 31-42.

Gajardo Jauregui, J. (2015). Vejez y soledad: Implicancias a partir de la construcción de la noción de riesgo. Acta Bioethica, 21, 199-205.

Galicia, E., Almeida, N. M. de y Carvalho, A. L. de (2020). La viudez en las mujeres. Aproximaciones diversas en las ciencias sociales. Revista Estudios Feministas, 28(2), e60544. https://doi.org/10.1590/18069584-2020v28n260544

Garay, S., Monteiro, L., Carbajal, M., Bonilla, R., Maciel, C. y Martínez, F. (2021). Soledad social en personas mayores en hogares unipersonales: El caso de Durango y Montevideo. En C. Arroyo (coord.), Las soledades en la vejez. Experiencias, significados y afrontamientos (pp. 31-56). Universidad Juárez del Estado de Durango.

Garza-Sánchez, R. I., González-Tovar, J., Rubio-Rubio, L. y Dumitrache-Dumitrache, C. G. (2020). Soledad en personas mayores de España 
y México: Un análisis comparativo. Acta Colombiana de Psicología, 23(1), 106-116.

Golden, J., Conroy, R. M., Bruce, I., Denihan, A., Greene, E., Kirby, M. y Lawlor, B. A. (2009). Loneliness, social support networks, mood and wellbeing in community-dwelling elderly. International Journal of Geriatric Psychiatry, 24(7), 694-700. https://doi.org/10.1002/gps.2181

Hacihasanoğlu, R. Yildirim, A. y Karakurt, P. (2012). Loneliness in elderly individuals, level of dependence in activities of daily living (ADL) and influential factors. Archives of Gerontology and Geriatrics, 54, 61-66.

Heylen, L. (2010). The older, the lonelier? Risk factors for social loneliness in old age. Ageing and Society, 30(7), 1177-1196. https://doi.org/10.1017/ S0144686X10000292

Hierro, G. (2003). La ética del placer. Universidad Nacional Autónoma de México.

Iglesias de Ussel, J., López, J., Díaz, M. P., Alemán, C., Trinidad, A. y Castón, P. (2001). La soledad en las personas mayores. Influencias personales, familiares y sociales. Análisis cualitativo. IMSERSO.

Instituto Nacional de Estadística (2019). Encuesta Continua de Hogares (ECH). https://www.ine.es/dynt3/inebase/index.htm?type=pcaxis\&path $=/ \mathrm{t} 20 / \mathrm{p} 274 / \mathrm{serie} /$ prov $/ \mathrm{p} 01 \&$ file $=$ pcaxis $\& \mathrm{~L}=0 \& \mathrm{dh}=0 \&-$ capsel $=0$

Instituto Nacional de Estadística (2020). Cifras de población. Datos provisionales al 1 de enero de 2020. https://www.ine.es/dyngs/INEbase/ es/operacion.htm?c=Estadistica_C\&cid=1254736176951\&menu=ultiDatos\&idp=1254735572981

Instituto Nacional de Estadística y Geografía (2017). Encuesta Nacional de los Hogares (ENH) 2017. https://www.inegi.org.mx/programas/enh/2017/

Instituto Nacional de Estadística y Geografía (2018). Encuesta Nacional de la Dinámica Demográfica 2018. https://www.inegi.org.mx/programas/ enadid/2018/

Instituto Nacional de Estadística y Geografía (2020). Censo de población y vivienda 2020. https://www.inegi.org.mx

Jelin, E. (1989). El celibato, la soledad y la autonomía personal: Elección personal y restricciones sociales. Estudios demográficos y urbanos, $4(1), 117-138$.

Lagarde, M. (2012). La soledad y la desolación. Consciencia y diálogo. Anales sobre temas de Ciencias Humanas, 3(3), 194-204. 
Lagarde, M. (2014). Los cautiverios de las mujeres. Madresposas, monjas, presas, putas y locas. Siglo XXI Editores, Universidad Nacional Autónoma de México.

López, J. y Díaz, M. del P. (2018). El sentimiento de soledad en la vejez. Revista Internacional de Sociología, 76(1), e085. https://doi.org/10.3989/ ris.2018.76.1.16.164

Matijasevic, M. T. y Ruiz, A. (2013). La construcción social de lo rural. Revista Latinoamericana de Metodología de la Investigación Social (5), 24-41. http://relmis.com.ar/ojs/index.php/relmis/issue/view/ busquedas_multiples $/ 15$

Martínez, R. y Celdrán, M. (2019). La soledad no tiene edad. Explorando vivencias multigeneracionales. Observatorio de la soledad, Amics de la gent gran.

Montes de Oca, V. (2003). Redes comunitarias, género y envejecimiento. Cuadernos de Investigación. Instituto de Investigaciones Sociales, Universidad Nacional Autónoma de México.

Montes de Oca, V. (2011). Viudez, soledad y sexualidad en la vejez: Mecanismos de afrontamiento y superación. Revista Kairós: Gerontología, 14(35),73-107.

Padilla Arroyo, A. (2006). Memoria y vivencias de la muerte y la orfandad. Secuencia: revista de historia y ciencias sociales, (66), 65-88.

Pinazo, S. y Bellegarde, M. (2018). La soledad de las personas mayores. Conceptualización, valoración e intervención. Fundación Pilares.

Qualter, P., Brown, S. L., Rotenberg, K. L., Vanhalst, R. A., Harris, R. A., Goossens, L., Bangee, M. y Munn, P. (2013). Trajectories of loneliness during childhood and adolescence: Predictors and health outcomes. Journal of Adolescence, 36(6), 1283-1293.

Ramos, M. (2017). Envejecer siendo mujer. Bellaterra.

Rubio, R. (2011). La influencia de la soledad en las formas de vida y convivencia de los mayores. En Instituto de Mayores y Servicios Sociales, Libro blanco del envejecimiento activo, IMSERSO.

Rubio, R., Cerquera, A. M., Muñoz, R. y Pinzón, E. A. (2011). Concepciones populares sobre soledad de los adultos mayores de España y Bucaramanga, Colombia. Diversitas: Perspectivas en Piscología, 7(2), 307-319.

Rueda, J. D. (2018). La soledad de las personas mayores en España. Una realidad invisible. Actas de Coordinación Sociosanitaria, (23), 43-64.

Scott, A. y Wenger, C. (1996). Género y redes de apoyo en la vejez. En S. 
Arber y J. Ginn (eds.), Relación entre género y envejecimiento. Enfoque sociológico (pp. 221-240). Narcea.

Sequeira Daza, D. (2011). La soledad en las personas mayores: Factores protectores y de riesgo. Evidencias empíricas en adultos mayores chilenos [Tesis doctoral]. Universidad de Granada.

Shiovitz-Ezra, S. y Leitsch, S. (2010). The role of social relationships in predicting loneliness: The national social life, health, and aging project. Social Work Research, 34(3), 157-167.

Varley, A. y Blasco, M. (2001). ¿Cosechan lo que siembran? Mujeres ancianas, vivienda y relaciones familiares en el México urbano. En C. Gomes (coord.), Procesos sociales, población y familia. Alternativas teóricas y empíricas en las investigaciones sobre vida doméstica (pp. 301-322). FLACSO, Miguel Ángel Porrúa.

Weiss, R. S. (1973). Loneliness: The experience of emotional and social isolation. MIT Press. https://psycnet.apa.org/record/1974-22306-000

Yuni, J. A. y Urbano, C. (2008). Envejecimiento y género: Perspectivas teóricas y aproximaciones del envejecimiento femenino. Revista Argentina de Sociología, 6(10), 151-169. 